\title{
Wireless Isometric Force Sensor Platform for Bedside Automated Evaluation and Therapeutics of Orofacial and Hand Muscle Systems Across the Lifespan
}

\author{
Jacob Greenwood*, Mohsen Hozan and Steven M Barlow \\ University of Nebraska, USA \\ *Corresponding author: Jacob Greenwood, Department of Biological Systems Engineering, University of Nebraska, \\ Lincoln 68583, USA
}

\section{ARTICLE INFO}

Received: 慧 September 09, 2019

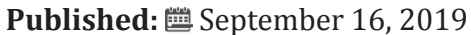

Citation: Jacob Greenwood, Mohsen Hozan, Steven M Barlow. Wireless Isometric Force Sensor Platform for Bedside Automated Evaluation and Therapeutics of Orofacial and Hand Muscle Systems Across the Lifespan. Biomed J Sci \& Tech Res 21(3)-2019. BJSTR. MS.ID.003591.

\begin{abstract}
A wireless biomechanical sensing technology, known as ForceWIN10, was developed and tested in our laboratory to provide clinicians and researchers with an integrated solution to conduct real-time bedside diagnostics and neurotherapeutics of muscle force dynamics among key motor control systems (i.e., hand-digits, orofacial) suitable for a wide range of clinical populations across the lifespan who have sustained injuries (i.e., cerebrovascular stroke, traumatic brain injury) or manifest progressive neuromotor disease (i.e., Parkinson's disease, amyotrophic lateral sclerosis, etc.). ForceWIN10 provides wireless sensor technology and data acquisition, custom test protocol creation, visuomotor stimulus control, analysis of isometric force signals, and file and database management.
\end{abstract}

\section{Introduction}

Accurate diagnosis and retraining are critical for rehabilitation of limb and orofacial muscle control systems in neuromotor disease (e.g., brain stroke, Parkinson's disease) and injury (e.g. traumatic brain injury). Impaired regulation of fine force dynamics underlies movement disorders associated with these neurological conditions [1-4]. For example, sensorimotor dysfunction during intricate movements of the face and hand is often prominent in post-stroke survivors, as brain lesions that damage cortical upper motor neurons also degrade motor units where lower motor neurons control striated muscle, particularly in high density, monosynaptic motor pools that project to muscles of the lower face and distal extremities of the limbs (hands, fingers, feet, etc.) [5-9]. Parkinson's disease, which is a progressive neuromotor disorder resulting from a depletion of dopaminergic neurons in the substantia nigra pars compacta, disrupts voluntary and postural force dynamics throughout the skeletal musculature, including the limbs (handdigit manipulation), chest wall (speech and metabolic breathing kinematics), and orofacial-laryngeal and aerodigestive motor systems involved in speech, song, and deglutition. Precise stiffness regulation for postural motor control is also significantly disrupted in basal ganglia disease $[4,10,11]$. The importance of active muscle force and stiffness regulation for fine motor control of distal extremities and orofacial structures underscores the need and accessibility to biomechanical measures for objective assessment of residual neuromotor capacity in health and disease [3,12-14].

Traditional, laboratory-based biomechanical (force) recording systems have relied on wired strain gage or force plate transduction systems conditioned by bridge amplifiers (analog or digital) with output signals digitized using an A/D converter (external box or internal plug-in module) interfaced to a benchtop computer. Device calibration usually involves the application of known loads (certified masses) to the various sensors to establish their gains and control for baseline offsets. Analysis of acquired data usually involves separate offline routines coded in a variety of languages. These laboratory setups have provided invaluable data and important contributions to our understanding of biomechanics in neurotypical and disordered participants. However, instrument size, cost, and portability generally limit where biomechanical measurements can be made, especially in challenging medical settings. 
ForceWIN10 alleviates the limitations inherent to most laboratory-based biomechanical recording and analysis systems, by making it possible to conduct bedside biomechanical assessments of hand and orofacial muscle systems. ForceWIN10 platform features custom designed Bluetooth wireless strain gage force sensors for the digits (hand) and several orofacial muscle systems (upper lip, lower lip, left and right oral angle, anterior tongue, and the jaws). This platform provides individualized biomechanical assessment of isometric force dynamics and can be configured for neurotherapeutic (biofeedback) retraining of an individual's affected muscle systems at bedside in acute or chronic care or rehabilitation settings. Translational research opportunities are likely to benefit from such an integrated biomechanics measurement technology, especially as it relates to cortical plasticity in survivors of brain stroke, traumatic brain injury, or progressive neuromotor disease.

\section{Materials and Methods}

The ForceWIN10 software was designed to provide exceptional control to the clinician while remaining easy to use. Device management, force test protocol creation, data collection, and dependent measure calculations can be performed within a single user-friendly program.

\section{Device Management}

Devices can be connected, calibrated, and configured without leaving the software. Wireless connectivity is provided via Bluetooth. A two-point calibration can be performed using any desired force (typically 0 and 2 newtons). The two reference forces are applied to a given transducer and digitized. The software then adjusts all future data points from the transducer with a linear calibration.

\section{Protocol Creation}

Custom protocols are generated and stored within the system. A clinician can create a wide range of visuomotor tracking tasks including the specification of absolute force level during rampand-hold, steps, triangular, or other arbitrary force targets. Multiple versions of a protocol can be stored and edited to allow randomization. These protocols can be used for assessment as well as for retraining therapy.

\section{Data Collection}

Data is collected interactively from the subject in real-time with biofeedback. A common visuomotor tracking paradigm, known as a ramp-and-hold isometric contraction task, is illustrated in this report and consists of a static target force level (e.g. $2 \mathrm{~N}$ ) which the subject attempts to attain as rapidly and accurately as possible [3,15-17]. The subject's force "ramps" from baseline to the target and then "holds" at the target until the end of the trial (typically 5 seconds). Subjects are instructed to relax after each trial. Each trial is followed by a delay of random duration, between 0.5 to 3 seconds, to minimize anticipatory behavior. To minimize the effects of thermal drift or postural changes, baseline correction can be performed between trials by clicking the designated button on the interface.

\section{Dependent Measure Calculations}

Various dependent measures can be calculated on any recorded session. The ForceWIN10 analysis routines can generate several dependent measures of force dynamics with the ability to export features for advanced analysis with statistical software or custom MATLAB scripts for aggregated analyses and visualizations across large number of subjects.

\section{System Overview}

The physical components of ForceWIN10 were designed to be accurate, compact, and low power. These components consist of a wireless data acquisition (DAQ) circuit, custom-designed and machined interchangeable stainless-steel fixtures, and a personal computer (PC) operating Microsoft Windows 10. The wireless DAQ collects, packetizes, and transmits samples using a Bluetooth Low Energy radio, two-stage amplifier, and 24-bit ADC. Since strain gage signals are in the microvolt range, the largest degradation of signalto-noise ratio is due to the noise of the first amplifier.

To increase the SNR, a low-noise amplifier (35nV/sqrtHz, 83dB CMRR) is used as the first stage of amplification. The amplified signal is band-limited to reduce noise and prevent aliasing in the ADC. The samples are then digitized at 120 samples per second by a 24-bit ADC with an integrated amplifier. The samples are packetized in groups of 4 to minimize packet overhead and are sent to the PC via Bluetooth. Power efficiency was a priority in component selection and firmware design; Current consumption was $0.78 \mathrm{~mA}$ at idle and $4.2 \mathrm{~mA}$ during active operation, with an additional $4.7 \mathrm{~mA}$ for half-bridge or 9.4 mA for full-bridge strain gage circuits. A small $400 \mathrm{mAh}$ single-cell LiPo battery (3.7V nominal), provides over 500 hours of standby time and 29 hours of continuous operation.

\section{Jaw Dynamics}

Jaw measurements are collected from the orofacial transducer base (Figure 1b \& 1c) which serves as the platform for all orofacial measurement paradigms. Two flat stainless-steel bars extend from a central structure terminating in an attachment point for a pair of 3D-printed titanium trays. One of the flat bars is instrumented with four strain gages in a full-bridge configuration to measure forces exerted between the maxilla and mandibular dentition. Multiple tray sizes are available to accommodate a wide range of maxillary and mandibular dentition sizes and bites, allowing a comfortable fit for most subjects above 6 years of age. The trays are impregnated with a polyvinylsiloxane impression material (Kerr Extrude XP, Kerr Corporation, Romulus, MI, USA) to create a custom mold (2-minutes set time) that results in a comfortable and stable connection to a subject's teeth (or alveolar surfaces for edentulous subjects).

\section{Orofacial Dynamics}

Lip, tongue, and oral angle force dynamics collection is identical except for placement and fixture shape. Lip measurements are 
transduced by a beam attached to a height adjustment fixture that is secured to the orofacial transducer base with a thumbscrew (Figure 1a \& 1c). The same height adjustment mechanism fixes the tongue transducer in place in between the dental trays (Figure 1e \& 1f). Oral angle force dynamics also use the height adjustment fixture along with an anterior-posterior and a medial-lateral adjustment fixture (Figure 1g \& 1h). The latter fixture also includes the instrumented cantilever. Each transducer has enough adjustment to account for variations in subject anatomy. Two strain gages are secured on each force cantilever (1 strain gage per side) in a halfbridge configuration.

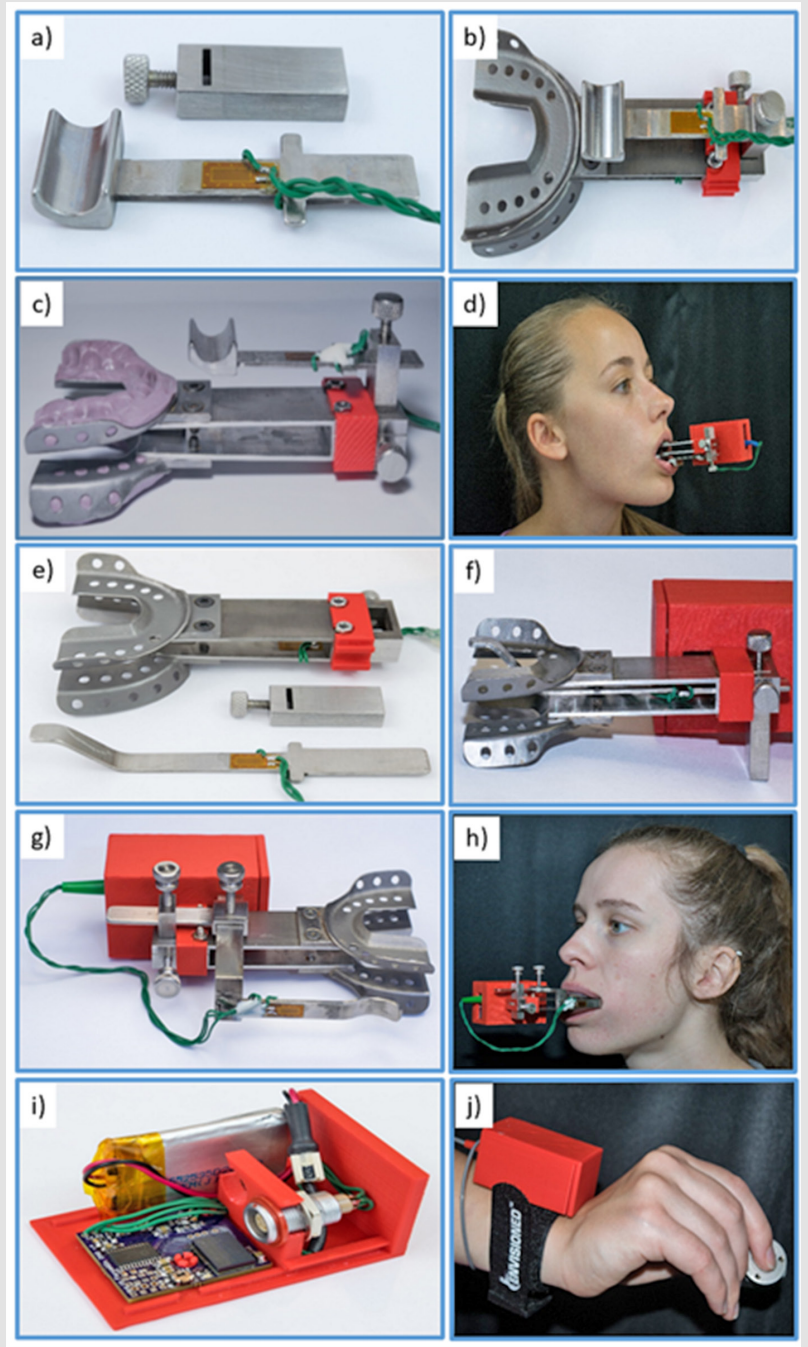

Figure 1: Instrumented orofacial transducer base, including

a) Lip force cantilever with strain gages, and slotted fixture for anterior-posterior and inferior-superior beam translation.

b) Lip force cantilever mounted to jaw force sensor platform with 3D-printed titanium maxillary and mandibular dental trays.

c) Side view of jaw and lip force sensors with polyvinylsiloxane dental impression molds.

d) Lower lip force sampling in situ with Bluetooth module.

e) An exploded view of tongue force cantilever with 2-arm strain gage application, slotted fixture for anterior-posterior and inferior-superior beam translation, and the interdental jaw yoke.

f) Assembled configuration for sampling anterior (lingual-alveolar) tongue isometric compression lip force.

g) Oral angle transducer assembly, including superior-inferior, anterior-posterior, and medial-lateral adjustment fixtures with instrumented force cantilever.

h) Oral angle force measurement in situ.

i) Thumb-index finger pinch force transduction, including Bluetooth wireless and signal conditioning module with power regulator, 24-bit DAC, and rechargeable Li-ion battery.

j) Test configuration with Bluetooth module and thumb-index finger pinch force load cell. 


\section{Digit Dynamics}

Finger measurements are collected using an off-the-shelf load cell (Cooper Instruments, Model LKCP 410-25 lb; Warrenton, VA, USA) that uses a full-bridge strain gage circuit to measure thumbfinger pinch forces up to $111.2 \mathrm{~N}$ (Figure 1j).

\section{Verification and Testing}

Verifying the ForceWIN10 system consisted of accuracy testing and an initial study in neurotypical humans. Accuracy was verified for each transducer by applying a known force, recording the measured force for multiple levels, and calculating the full-scale percent error as well as the R-squared linear fit coefficient. A preliminary study was conducted to show the ability of the device to work in a clinical setting and collect signals at least as good as found in the current literature. The study consisted of ten ramp-and-hold isometric sequences at $0.25,0.50,1.00$, and $2.00 \mathrm{~N}$ in a repeated measure, randomized block design. The full study is described in a separate paper with $\mathrm{N}=40$ young adults [3]. For the present report, a neurotypical 21-year-old male is examined along with summative statistics over the group.

\section{Subjects}

Forty (40) neurotypical adults (20F/20M, 22.23 [SD = 2.07] years old) were recruited regardless of ethnicity or race. Written informed consent, approved by the University of Nebraska Institutional Review Board, was obtained for each test subject. The inclusion criteria consisted of right-handed adults with no report of neurological illness or injury, not taking regular medication, and with normal corrected vision. Subjects with neurological, sensory or muscular deficits, trauma to face or hand and fingers, or with abnormal skin sensitivity on face or hand were excluded from this study. Subjects were compensated for their participation in this study.

\section{Result and Discussion}

The worst full-scale error in the calibrated range of any transducer was $3.32 \%$ for the lip transducer at $2 \mathrm{~N}$, however, the average full-scale error was $0.64 \%$. When looking at linearity, the worst linearity was the tongue transducer with an R-squared value of 0.9994 . The jaw, lip, and finger transducers had linearity values of $1.0000,0.9994$, and 0.9996 respectively. The first attempt at calculating the accuracy and linearity of these sensors showed a thermal drift during a warm-up period. After waiting approximately 5 minutes, the drift was no longer observed, and the accuracy measurements could take place. The high linearity of these transducers suggests that the majority of the deviations can be attributed to calibration error rather than non-linearity or drift.

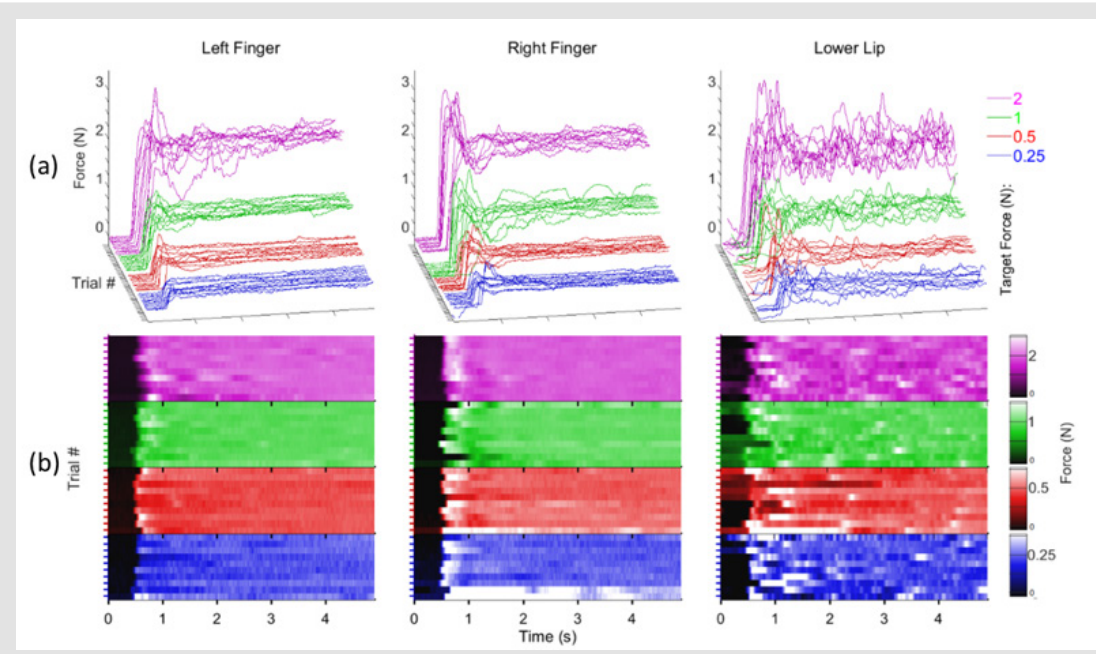

Figure 2:

a) Individual ramp-and-hold force trials at 0.25 (blue), 0.5 (red), 1.0 (green), and $2.0 \mathrm{~N}$ (purple) target force levels in waterfall display format for the right thumb-index finger, left thumb-index finger, and lower lip sampled from a 21 year old neurotypical male.

b) absolute force amplitude heat map depicting onsets and hold-phase contractions as a function of structure and target for a single participant.

A representative set of ramp-and-hold force waveforms sampled from a 21-year old male is shown from the right thumbindex finger, left thumb-index finger, and lower lip at four target force levels (Figure 2a). Individual contractions, sequenced along the z-axis in each waveform display, reveal the initial baseline 'reaction time interval' followed by the rapid force recruitment to peak, and the subsequent hold-phase to contraction termination (5 sec). Increased variability in isometric force onsets and holdphase performance, and a shorter reaction time for the lower lip trials compared to the thumb-index fingers were observed (Figure 2b). Examination of the mean and standard deviation of hold-phase isometric performance at various target forces for 20 males and 20 females (Figures $3 \& 4$ ), show the data are normally distributed about the mean with a small standard deviation. A 40-trial rampand-hold force dynamics assessment plus 3 maximum voluntary contractions can be completed for a single muscle system in approximately 7 minutes. 


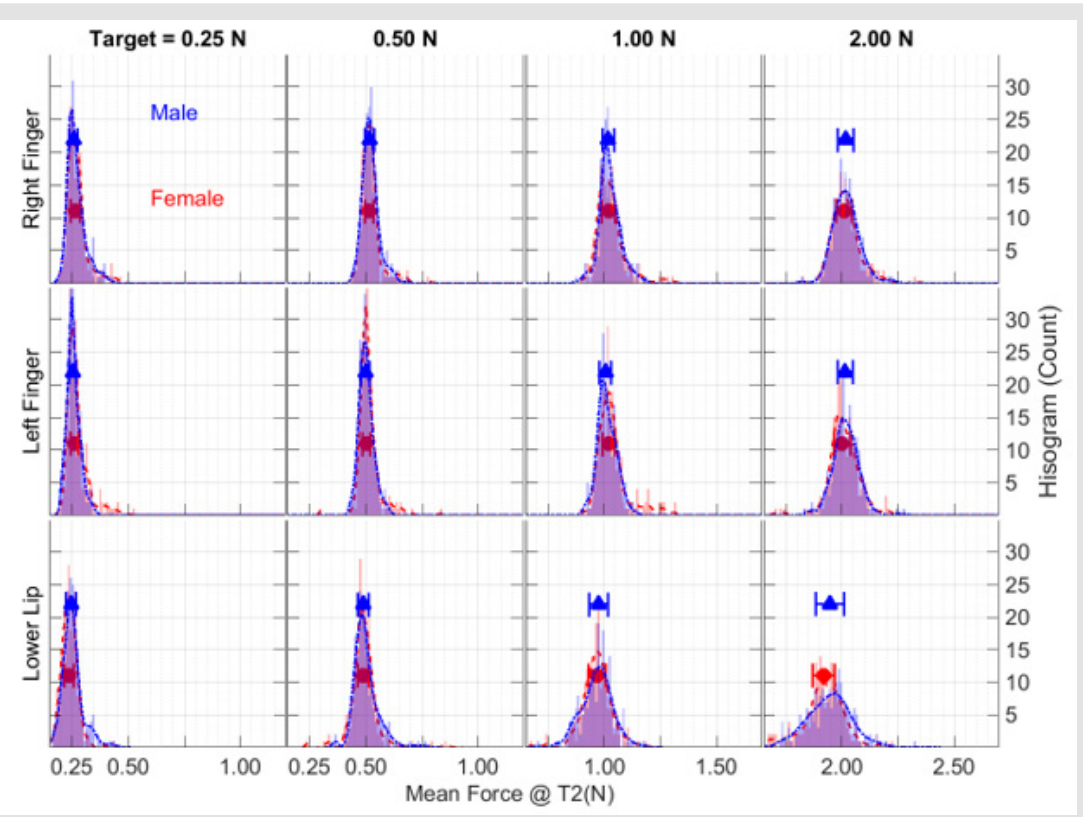

Figure 3: Total sample distributions of mean force during the hold-phase for 20 male (blue) and 20 female (red) participants as a function of target force and test structure plotted as frequency histograms along with the median \pm median absolute deviation (MAD) symbols and error bars.

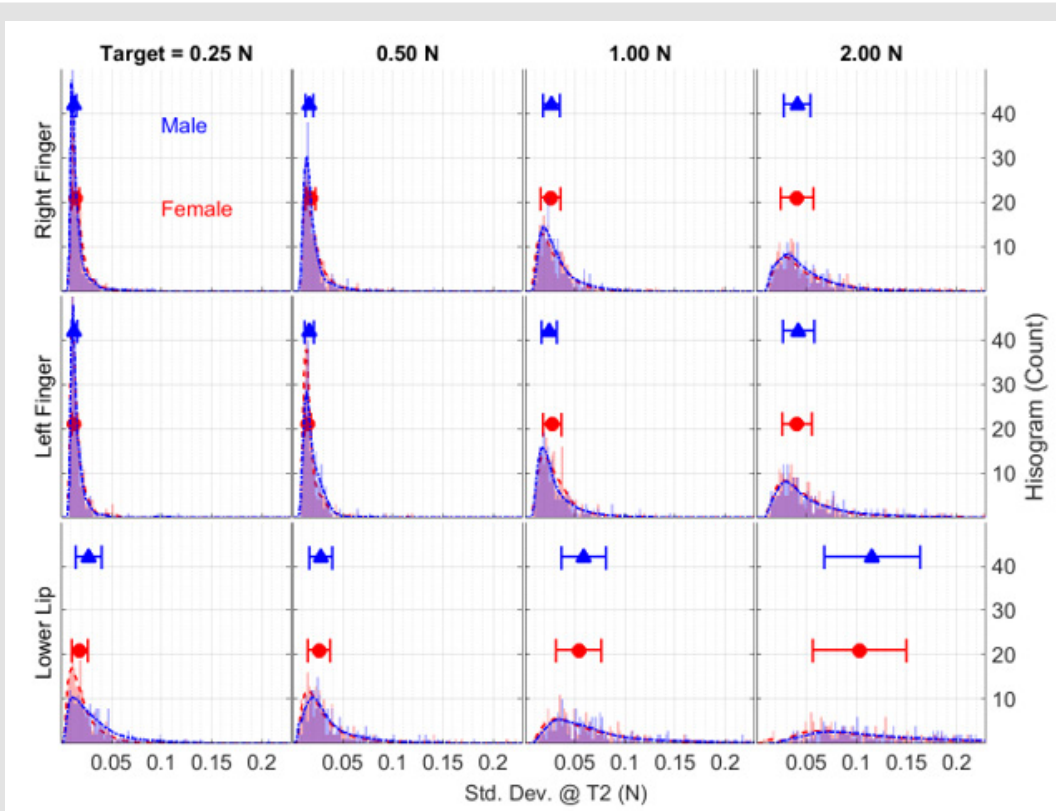

Figure 4: Total sample distributions of the standard deviation of isometric force during the hold-phase for 20 male (blue) and 20 female (red) participants as a function of target force and test structure plotted as frequency histograms along with the median \pm median absolute deviation (MAD) symbols and error bars.

\section{Conclusion}

The ForceWIN10 platform described in the present report offers clinicians and scientists a portable, wireless, and versatile tool for measurement of isometric force dynamics in orofacial and hand/digit muscle systems. ForceWIN10 has already been used to generate a neurotypical database in young adults [3], with ongoing studies in children (ages 7-12 years) [19], and older adults (35-70 years) to characterize fine force regulation and maximum voluntary force generation across the lifespan. A small database of orofacial and thumb-index force dynamics has also been collected in patients who have sustained unilateral middle cerebral artery infarcts resulting from ischemic stroke with promising results.

Improved portability and operational ease of biomechanical assessment instruments will result in broader clinical use in patients with movement disorders [3,15,17-20]. The inclusion of such biomechanical measures is expected to promote individualized treatment programs to support the rehabilitation of orofacial and hand-digit motor skills which are often impaired in neuromotor disease and injury. 


\section{Data Availability}

The data used to support the findings of this study are available from the corresponding author upon request.

\section{Conflicts of Interest}

The authors declare that there is no conflict of interest regarding the publication of this paper.

\section{Funding Statement}

This study was supported in part by the Nebraska Research Initiative (Barlow), and the Barkley Trust Foundation (Barlow).

\section{Acknowledgement}

Approval for this study was given by the University of Nebraska Institutional Review Board (IRB\#16151). Gratitude is expressed to Brianna Wardyn, Kaytlin Tippin, and Anna Jean Scarborough for technical and administrative support.

\section{References}

1. Kang N, Cauraugh JH (2015) Force control in chronic stroke. Neuroscience \& Biobehavioral Reviews 52: 38-48.

2. Dias CP, Freire B, Goulart NBA, Onzi, ES, Becker J, et al. (2017) Muscle architecture and torque production in stroke survivors: an observational study. Topics in Stroke Rehabilitation 24(3): 206-213.

3. Barlow SM, Hozan M, Lee J, Greenwood J, Custead R, et al. (2018) Orofacial and thumb-index finger ramp-and-hold isometric force dynamics in young neurotypical adults. Journal of Biomechanics 72:81-89.

4. Chu SY, Barlow SM, Lee J (2015) Face-Referenced Measurement of Perioral Stiffness and Speech Kinematics in Parkinson's Disease. Journal of Speech, Language, and Hearing Research 58(2): 201-212.

5. Gemperline JJ, Allen S, Walk D, Rymer WZ (1995) Characteristics of motor unit discharge in subjects with hemiparesis. Muscle \& Nerve 18(10): 1101-1114.

6. Frontera WR, Grimby L, Larsson L (1997) Firing rate of the lower motoneuron and contractile properties of its muscle fibers after upper motoneuron lesion in man. Muscle \& Nerve 20(8): 938-947.

7. Hara Y, Masakado Y, Chino N (2004) The physiological functional loss of single thenar motor units in the stroke patients: when does it occur? Does it progress? Clinical Neurophysiology: Official Journal of the International Federation of Clinical Neurophysiology 115(1): 97-103.

8. Lukács M (2005) Electrophysiological signs of changes in motor units after ischaemic stroke. Clinical Neurophysiology: Official Journal of the
International Federation of Clinical Neurophysiology 116(7): 15661570.

9. Schimmel M, Leemann B, Schnider A, Herrmann FR, Kiliaridis S, et al. (2013) Changes in oro-facial function and hand-grip strength during a 2-year observation period after stroke. Clinical Oral Investigations. 17(3): 867-876.

10. Hunker CJ, Abbs JH, Barlow SM (1982) The relationship between parkinsonian rigidity and hypokinesia in the orofacial system: A quantitative analysis. Neurology 32(7): 749-749.

11. Chu SY, Barlow SM, Kieweg D, Lee J (2010) Oro STIFF: Face-referenced measurement of perioral stiffness in health and disease. Journal of Biomechanics 43(8): 1476-1482.

12. Barlow SM (1998) Real time modulation of speech-orofacial motor performance by means of motion sense. Journal of Communication Disorders 31(6): 511-534.

13. Cole KJ (2015) Hand motor control: maturing an immature science. Motor Control 19(2): 131-134.

14. Latash ML, Friedman J, Kim SW, Feldman AG, Zatsiorsky VM (2010) Prehension synergies and control with referent hand configurations. Experimental Brain Research 202(1): 213-229.

15. Seo NJ, Enders LR, Motawar B, Kosmopoulos ML, Fathi-Firoozabad M (2015) The extent of altered digit force direction correlates with clinical upper extremity impairment in chronic stroke survivors. Journal of Biomechanics 48(2): 383-387.

16. Schimmel M, Ono T, Lam OLT, Müller F (2017) Oro-facial impairment in stroke patients. Journal of Oral Rehabilitation 44(4): 313-326.

17. Barlow SM, Burton MK (1990) Ramp-and-Hold Force Control in the Upper and Lower Lips. Journal of Speech, Language, and Hearing Research 33(4): 660-675.

18. Hoffman E (2018) Orofacial and hand force dynamics in neurotypical children. University of Nebraska-Lincoln, Lincoln, NE.

19. Coronado JL, Ulloa Perez A, Garcia Cordova F, Villalba Fernandez JJ (1999) Experimental development for thumb-index pinch force measurements during object manipulation with application to robotic hands. IEEE SMC'99 Conference Proceedings. 1999 IEEE International Conference on Systems, Man, and Cybernetics (Cat. No.99CH37028) 6: 786-791.

20. Dobkin BH (2005) Clinical practice. Rehabilitation after stroke. The New England Journal of Medicine 352(16): 1677-1684.

21. Hsu HY, Lin CF, Su FC, Kuo HT, Chiu HY, et al. (2012) Clinical application of computerized evaluation and re-education biofeedback prototype for sensorimotor control of the hand in stroke patients. Journal of Neuroengineering and Rehabilitation 9: 26

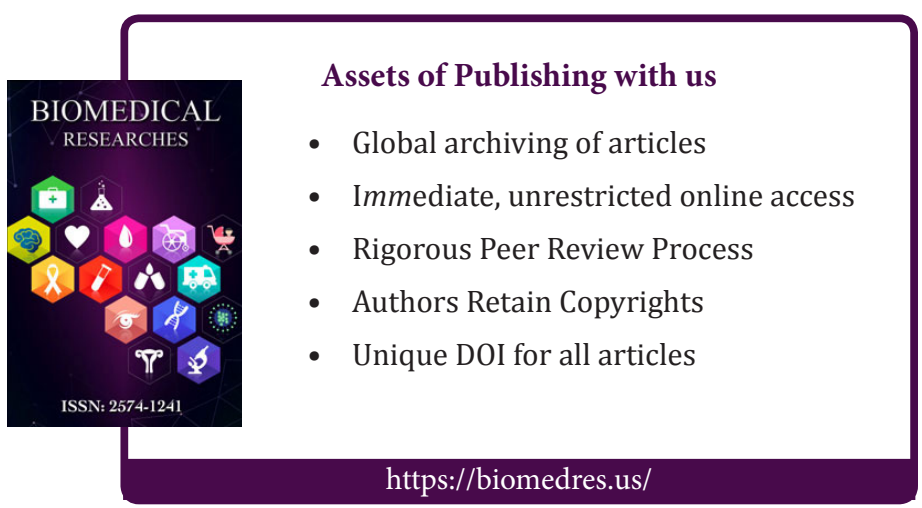

ISSN: 2574-1241

DOI: 10.26717/BJSTR.2019.21.003591

Jacob Greenwood. Biomed J Sci \& Tech Res

This work is licensed under Creative Commons Attribution 4.0 License

Submission Link: https://biomedres.us/submit-manuscript.php

Copyright@ Jacob Greenwood | Biomed J Sci \& Tech Res | BJSTR. MS.ID.003591. 